\title{
La investigación en Seguridad y Salud Laboral en España. Estudio exploratorio y de casos
}

María del Carmen Pardo-Ferreira, Juan Carlos Rubio-Romero, Juan Antonio Torrecilla García, Manuel Suárez-Cebador, Domingo Pérez-Mira

Recibido: 5 de Marzo de 2018 / Aceptado: 3 de Abril de 2018

\section{Resumen}

Investigación, desarrollo tecnológico e innovación son uno de los pilares del crecimiento económico. Aunque las estrategias de seguridad y salud laboral inciden en la necesidad de impulsar la investigación en esta materia, existen pocas líneas de financiación específicas. Para fomentar el impulso es fundamental conocer la realidad de lo que se hace. Así, el presente estudio tiene por objetivo realizar un acercamiento al conocimiento del estado de la investigación, desarrollo e innovación, en esta materia en España. Los resultados indican una mayor cantidad de proyectos y financiación en materia de medio ambiente y calidad que en seguridad y salud laboral.

\section{Palabras clave}

Investigación, Desarrollo, Innovación, Seguridad y salud laboral, Estudio de casos.

\section{Introducción}

La investigación, el desarrollo tecnológico y la innovación (en adelante $\mathrm{I}+\mathrm{D}+\mathrm{i}$ ) son considerados como uno de los pilares fundamentales para el crecimiento económico (Greenhalgh y Rogers, 2010; Vives, 2008). Para potenciarlos y canalizarlos, tanto a nivel internacional como nacional y autonómico, se han desarrollado estrategias, marcos, estructuras y normas para su impulso. Así, a nivel comunitario la principal fuente de canalización de las actividades de I+D europeas se han llevado a cabo a través de los Programas Marco de la Unión Europea, actualmente a través del Programa Marco de Investigación e Innovación de la Unión Europea Horizonte 2020 (2014-2020).

A nivel nacional, anualmente, el Ministerio de Economía y Competitividad hace pública una convocatoria de ayudas para la realización de proyectos de investigación, desarrollo

\footnotetext{
María del Carmen Pardo-Ferreira * carmenpf@uma.es

Juan Carlos Rubio-Romero * juro@uma.es

Juan Antonio Torrecilla García * juantorrecilla@uma.es

Manuel Suárez-Cebador * suarez_c@uma.es

Domingo Pérez-Mira ** dperezmi@uoc.edu

* Escuela de Ingenierías Industriales, Dpto. de Economía y Administración de Empresas. Universidad de Málaga

** Doctor Ingeniero Industrial
}

e innovación, asociada al Plan Nacional de I+D+i. Actualmente disponemos de la Estrategia Española de Ciencia y Tecnología y de Innovación 2013-2020, dentro de la que está vigente el Plan Estatal de Investigación Científica y Técnica y de Innovación 2017-2020. La Estrategia Española es el instrumento marco en el que quedan establecidos los objetivos generales a alcanzar durante el período 2013-2020 ligados al fomento y desarrollo de las actividades de $\mathrm{I}+\mathrm{D}+\mathrm{i}$ en España. Estos objetivos se alinean con los que marca la Unión Europea dentro del programa marco para la financiación de las actividades de $\mathrm{I}+\mathrm{D}+\mathrm{i}$ «Horizonte 2020» para el período 2014-2020, contribuyendo a incentivar la participación activa de los agentes del Sistema Español de Ciencia, Tecnología e Innovación en el espacio europeo. Respecto a las convocatorias Retos-Colaboración del Programa Estatal de Investigación, Desarrollo e Innovación Orientada a los Retos de la Sociedad, el objetivo es el apoyo a proyectos en cooperación entre empresas y organismos de investigación, con el fin de promover el desarrollo de nuevas tecnologías, la aplicación empresarial de nuevas ideas y técnicas, y contribuir a la creación de nuevos productos y servicios.

Por otra parte, el Centro para el Desarrollo Tecnológico Industrial (CDTI) es una Entidad Pública Empresarial, que promueve la innovación y el desarrollo tecnológico de las empresas españolas. Pese a centrar sus actividades más propiamente en la empresa que en la investigación desde la Universidad, entre sus actividades se encuentra la de promover la inter-fase del Sistema español de Ciencia-Tecnología-Empresa, así como la de promover la participación en Programas Europeos tanto de empresas como de otras entidades de investigación. Igualmente, la Fundación Española para la Ciencia y la Tecnología (FECYT), creada en 2001 con el objeto de prestar un servicio continuado y flexible al sistema es- 
pañol de Ciencia-Tecnología-Empresa, pretende convertirse en plataforma de encuentro, análisis y debate interdisciplinar e intersectorial para las comunidades científica, tecnológica y empresarial del país. Para todos estos fines FECYT pone en marcha convocatorias para la concesión de ayudas ligadas a estas actividades.

Las Administraciones Autonómicas suelen contemplar partidas presupuestarias, más o menos significativas para el fomento de la investigación en sus respectivos territorios igualmente. Tampoco podemos olvidarnos de la relación directa Universidad-Empresa, aunque algunos programas estatales, autonómicos o europeos, se apoyan también en esta relación Universidad-Empresa a través de sus Oficinas de Transferencia de Resultados de Investigación (en adelante OTRIs), como se representa en la figura 1 de la triple hélice Universidad-Empresa.
Figura 1 Modelo triple hélice Universidad-Empresa

\section{Universidad-Empresa}

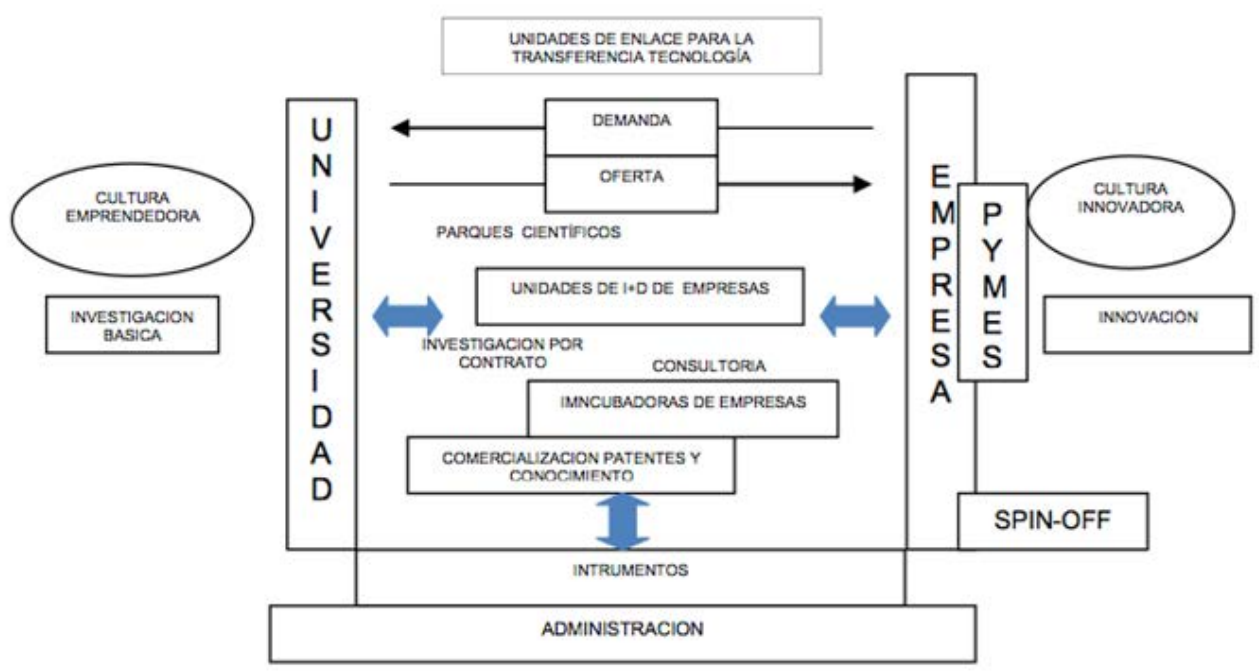

Sin duda la financiación de estos organismos es fundamental para el éxito de los proyectos de $\mathrm{I}+\mathrm{D}+\mathrm{i}$ (Blindenbach-Driessen y Van Den Ende, 2006; Buesa et al., 2006; Crow, 1988; Freeman y Soete, 2009; Hammadou et al., 2014; Santamaría et al., 2010; Tether, 2002). Así la falta de financiación es entendida como un obstáculo para la promoción de la $\mathrm{I}+\mathrm{D}+\mathrm{i}$ y se identifica la búsqueda de financiación como uno de los principales problemas para el desarrollo de dichos proyectos (Evangelista y Sirilli, 1998, Pereiras y Huergo, 2006).

El papel jugado por los anteriores organismos en relación a la cooperación entre empresas e instituciones y organismos públicos de investigación es también fundamental, en relación al éxito de la I+D+i (Arroyabe et al., 2015; Barajas y Huergo, 2006; Blindenbach-Driessen y Van Den Ende, 2006; Buesa et al., 2006; Crow, 1988; Freeman y Soete, 2009; Hottenrott y Lopes-Bento, 2014; Kingsley et al., 1996; Lhuillerya y Pfister, 2009; Santamaría et al., 2010; Santamaría y Rialp, 2007; Schlegelmilch y Chini, 2003; Tether, 2002). Así la elección del socio para los proyectos de cooperación está estrechamente relacionada con los objetivos del proyecto (Santamaría y Rialp, 2007; Quintana y Benavides 2010), siendo la cooperación vertical, entre empresas de diferentes sectores, la preferida cuando la empresa persigue objetivos comerciales; con universidades y centros tecnológicos cuando hay objetivos de financiación pública, además de potenciar las capacidades tecnológicas creadoras de innovaciones de alto valor en el mercado; y la cooperación con los com- petidores u horizontal cuando las empresas buscan socios con los que conseguir financiación para los proyectos de I+D+i (Belderbos et al., 2014).

En relación a la investigación específicamente en materia de seguridad y salud laboral, encontramos pocas líneas específicas de financiación. En las 3 prioridades del Programa Marco: "Retos Sociales", promover el "Liderazgo Industrial" en Europa, y reforzar la "Ciencia Excelente" de su base científica, no encontramos el tópico directamente, aunque indirectamente lo podemos considerar incluido en otros tópicos o de forma transversal. Así, la prioridad "Retos Sociales" incluye especialmente los retos que pueden tener relación tales como: 1 . Salud, cambio demográfico y bienestar, 4. Transporte inteligente, ecológico e integrado, y 5. Acción por el clima, medioambiente, eficiencia de los recursos y materias primas. A nivel de las comunidades autónomas a lo largo del tiempo hemos encontrado líneas específicas, normalmente dependientes directamente de las Consejerías de Trabajo, Empleo o similares, o de las Direcciones Generales de Trabajo o Seguridad y Salud Laboral.

A pesar de no contar con un tópico específico, en el ámbito de las estrategias de seguridad y salud laboral, tanto en el marco estratégico de la UE en materia de salud y seguridad en el trabajo 2014-2020 como en la propia Estrategia Española de Seguridad y Salud en el Trabajo 2015-2020, se incide en la necesidad de impulsar la investigación en materia de seguridad y salud laboral. Así, se identifica la necesidad 
de establecer una red de institutos públicos de investigación en seguridad y salud laboral, así como consolidar dicha investigación a través de ayudas públicas y la formación de investigadores de este campo, planteando acciones de mejora en cuanto a: evaluación de riesgos laborales, reducción de enfermedades relacionadas con el trabajo, prevención de nuevos riesgos o riesgos emergentes, etc.

La investigación en seguridad y salud no cabe duda que es un campo muy amplio, debido a la transversalidad y multidisciplinariedad, lo que dificulta focalizar su impulso. Así, la prevención de riesgos laborales incluye en su alcance "el bienestar social, mental y físico de los trabajadores" (OIT, 2017). Esto en la práctica significa que la investigación y desarrollo de nuevos productos químicos, materiales, equipos, sistemas, etc., pueden considerarse dirigidos a la seguridad y salud si de alguna forma contribuyen a esa mejora del bienestar social, mental y físico de una persona en su lugar de trabajo. Si además pensamos que casi cualquier lugar, es el lugar de trabajo para alguna persona, los límites pueden ser infinitos. La delimitación y la clasificación de los proyectos de $\mathrm{I}+\mathrm{D}+\mathrm{i}$ en seguridad y salud es por tanto harto complicada, pues en la práctica estos proyectos son abordados de manera holística (Rial-González et al., 2005).

Con el fin de poder impulsar adecuadamente la investigación en seguridad y salud laboral, es fundamental que conozcamos la realidad de lo que se hace al respecto, y sin embargo no existen investigaciones relacionadas. El presente estudio tiene por objetivo realizar un primer acercamiento al conocimiento del estado de la investigación, desarrollo e innovación, en materia de seguridad y salud en España. Para ello, se estudia durante el periodo 2009-2014 el número de proyectos, el grado de financiación, la tipología en cuanto a la cooperación, estrategias, motivación, etc., de forma comparada con los realizados en las áreas de medio ambiente y calidad, aspectos que tradicionalmente afines en relación a los sistemas de gestión integrados.

\section{Diseño de la investigación, metodolo- gía y materiales}

Fueron realizados dos análisis para alcanzar el objetivo propuesto. Por un lado, un estudio de la tipología y financiación de los proyectos financiados a partir de la información pública disponible. La finalidad de este análisis fue obtener la distribución de proyectos empresariales de I+D+i de Seguridad y Salud Laboral, tanto individuales como en cooperación, desarrollados frente a las áreas de Calidad y Medio Ambiente. Por otro lado, el segundo análisis consistió en un estudio de casos centrado en 3 empresas de diferentes sectores. Así se perseguía profundizar en los resultados y definir patrones de comportamiento. El periodo temporal analizado para ambos estudios fue de 2009 a 2014.

En relación al primer análisis de la información accesible de tipos de proyectos y financiación, se acudió a las fuentes de información pública en internet. A nivel nacional se analizaron los proyectos financiados por el Ministerio de Economía y Competitividad (en adelante MINECO) a través del Centro para el Desarrollo Tecnológico e Industrial (en adelante CDTI), el cual ejecuta las políticas del Gobierno en materia de investigación científica, el desarrollo tecnológico e innovación en todos los sectores. A nivel de comunidades autónomas se pudo acceder a la información básica de los proyectos financiados en Andalucía por la Agencia de Innovación y Desarrollo de Andalucía (en adelante IDEA), la Corporación Tecnológica de Andalucía (en adelante CTA), y en Galicia por la Agencia Gallega de Innovación (en adelante GAIN).

La clasificación de la diferente tipología de proyectos la realizó un investigador del equipo con más de 10 años de experiencia en el desarrollo de proyectos de $\mathrm{I}+\mathrm{D}+\mathrm{i}$ en las distintas áreas, aunque en algunas entidades ésta ya estaba realizada. Para ello, el investigador realizó la clasificación llevando a cabo un análisis sintáctico del título del proyecto basado en palabras clave, incluyendo sus sinónimos según el Diccionario de la Real Academia Española 2015 (García y Suárez, 2013). Una vez localizado en el texto la raíz de alguna de las palabras, el proyecto se seleccionó y clasificó en su área o áreas correspondientes. En los casos dudosos, el experto tuvo que decidir la tipología principal. Puesto que la tipología hubiera sido demasiado amplia si no se acotaba, se decidió estudiar los proyectos en materia de seguridad y salud en relación a los proyectos que podemos considerar afines siguiendo la tradición de los sistemas integrados de gestión de calidad, medio ambiente y seguridad y salud laboral. Las palabras clave pueden verse en la tabla 1. Así mismo, debido a la importancia de conocer si el proyecto se hizo en cooperación o de forma individual, esta información también se registró y analizó 
Tabla 1 Palabras claves utilizadas para la clasificación sintáctica de la tipología de los proyectos.

\begin{tabular}{ll}
\hline \multicolumn{1}{c}{ Área de clasificación } & \multicolumn{1}{c}{ Palabras clave } \\
\hline Medioambiente & $\begin{array}{l}\text { Medioambiente, ambiental, sostenibilidad, reciclaje, residuos, } \\
\text { ecológico, bio. }\end{array}$ \\
\hline Calidad & Calidad, mejora de procesos, control de calidad \\
\hline Seguridad y Salud Laboral & $\begin{array}{l}\text { Riesgos laborales, puesto de trabajo, seguridad y salud laboral, } \\
\text { prevención, ergonomía, enfermedad, accidente. }\end{array}$ \\
\hline
\end{tabular}

En relación al segundo análisis, el estudio de casos, hay que decir que esté método de investigación cualitativo es ampliamente utilizado en áreas complejas y cambiantes, sobre las que no existe un histórico representativo previo (Yin, 2009, Chaminade et al, 1999), como puede ser el objeto de esta investigación. El estudio de casos aportó una interpretación complementaria a los resultados cuantitativos obtenidos en el primer análisis (Patton, 2002). El uso combinado de ambos métodos mejoró la representatividad de los casos y respaldó cierta generalización (Seale y Silverman, 1997).

Para ello, se seleccionaron 3 empresas multinacionales que se encontraban se encontraban en continuo crecimiento y expansión, y que realizaban proyectos de $\mathrm{I}+\mathrm{D}+\mathrm{i}$ como herramienta de incremento de competitividad, y que además disponían de sistemas de gestión integrados de Seguridad y Salud Laboral, Calidad y Medio Ambiente. Se trata de tres empresas españolas privadas y con actividades en diferentes sectores: Energético, Obra Civil y Tecnologías de la Información y Comunicación.

Siguiendo las teorías de Yin (2009) para la elaboración de herramientas de los estudios de casos, se diseñó un protocolo de recogida de datos específico que permitió comparar los resultados y evitar al mismo tiempo los juicios de valor. Los datos fueron obtenidos de fuentes tanto cualitativas como cuantitativas, es decir, documentos publicados, registros de archivos, entrevistas directas con los responsables de $\mathrm{I}+\mathrm{D}+\mathrm{i}$ de las empresas, etc. (Chetty, 1996). El trabajo de campo se realizó durante 2015.

Mediante este estudio de casos, además de realizar una descripción de la empresa, entendiendo cuál era su actividad principal y las líneas de negocio de la misma, se analizó qué actividad se desarrollaba en $\mathrm{I}+\mathrm{D}+\mathrm{i}$ en Medio Ambiente, Calidad, y Seguridad y Salud Laboral, la financiación e inversión realizada en los proyectos $\mathrm{I}+\mathrm{D}+\mathrm{i}$, la estrategia e interés de la empresa en desarrollar dichos proyectos de I+D+i, la organización de la empresa para el desarrollo de los proyectos de I+D+i, la importancia de la Seguridad y Salud Laboral en la organización desde el punto de vista del desarrollo de actividades de $\mathrm{I}+\mathrm{D}+\mathrm{i}$, si los proyectos se realizaban de forma individual o en colaboración y el tipo de colaboración, así como los resultados obtenidos al respecto.

\section{Resultados y discusión}

\subsection{Resultados del estudio de la información publicada sobre la tipología de proyectos}

Fueron identificados 20.782 proyectos presentados en competencia para su financiación durante el periodo 20092014 en los organismos antes mencionados. De ellos, a 7.771 se les concedió financiación. Tras realizar la búsqueda de la información disponible sobre dichos proyectos, se accedió a los datos de 4.447 proyectos. Estos proyectos fueron el punto de partida para llegar a realizar el análisis cuantitativo de los proyectos de Seguridad y Salud Laboral de forma comparada con las áreas de Medio Ambiente y Calidad.

A continuación se procedió a clasificar los proyectos identificados conforme a las tres áreas de estudio y se encontró que muchos de los proyectos no se enmarcaban en ninguna de las tres áreas. Tan solo un $12.34 \%$ de los proyectos identificados quedaban incluidos en una o varias de las áreas de estudio. Así, la muestra final estuvo compuesta por 548 proyectos, de los cuales 482 eran desarrollados de forma individual y 66 en cooperación.

Estos proyectos fueron analizados cuantitativamente y se encontró que el organismo que más proyectos incentiva es CDTI, lo cual es coherente dado que la financiación y apoyo a los proyectos de $\mathrm{I}+\mathrm{D}+\mathrm{i}$ de empresas españolas es una de sus misiones principales. De hecho, hasta 2015 ha sido el principal agente en cuanto a la gobernanza de las políticas de $\mathrm{I}+\mathrm{D}+\mathrm{i}$ en España, en relación a su fomento y financiación (Myro y López, 2016). Actualmente, junto al CDTI se sitúa a la Agencia Estatal de Innovación que nació a finales de 2015. En general, los organismos regionales públicos y privados incentivan proyectos de menor alcance económico que CDTI, ya que gestionan menos fondos y el número de proyectos que incentivan es mayor proporcionalmente que en CDTI. Además, estos organismos incentivan muchos más proyectos individuales que en cooperación. Siendo el CDTI el que prácticamente define la tendencia y proporción de los proyectos analizados en éste ámbito. 
Figura 2 Distribución acumulada de proyectos analizados de $\mathrm{I}+\mathrm{D}+\mathrm{i}$ individuales de Seguridad y Salud Laboral, Calidad y Medio Ambiente de CDTI, CTA, IDEA y GAIN durante el período 2009 a 2014
Figura 3 Distribución acumulada de proyectos analizados de $\mathrm{I}+\mathrm{D}+\mathrm{i}$ en cooperación de Seguridad y Salud Laboral, Calidad y Medio Ambiente de CDTI, CTA y GAIN durante el período 2009 a 2014
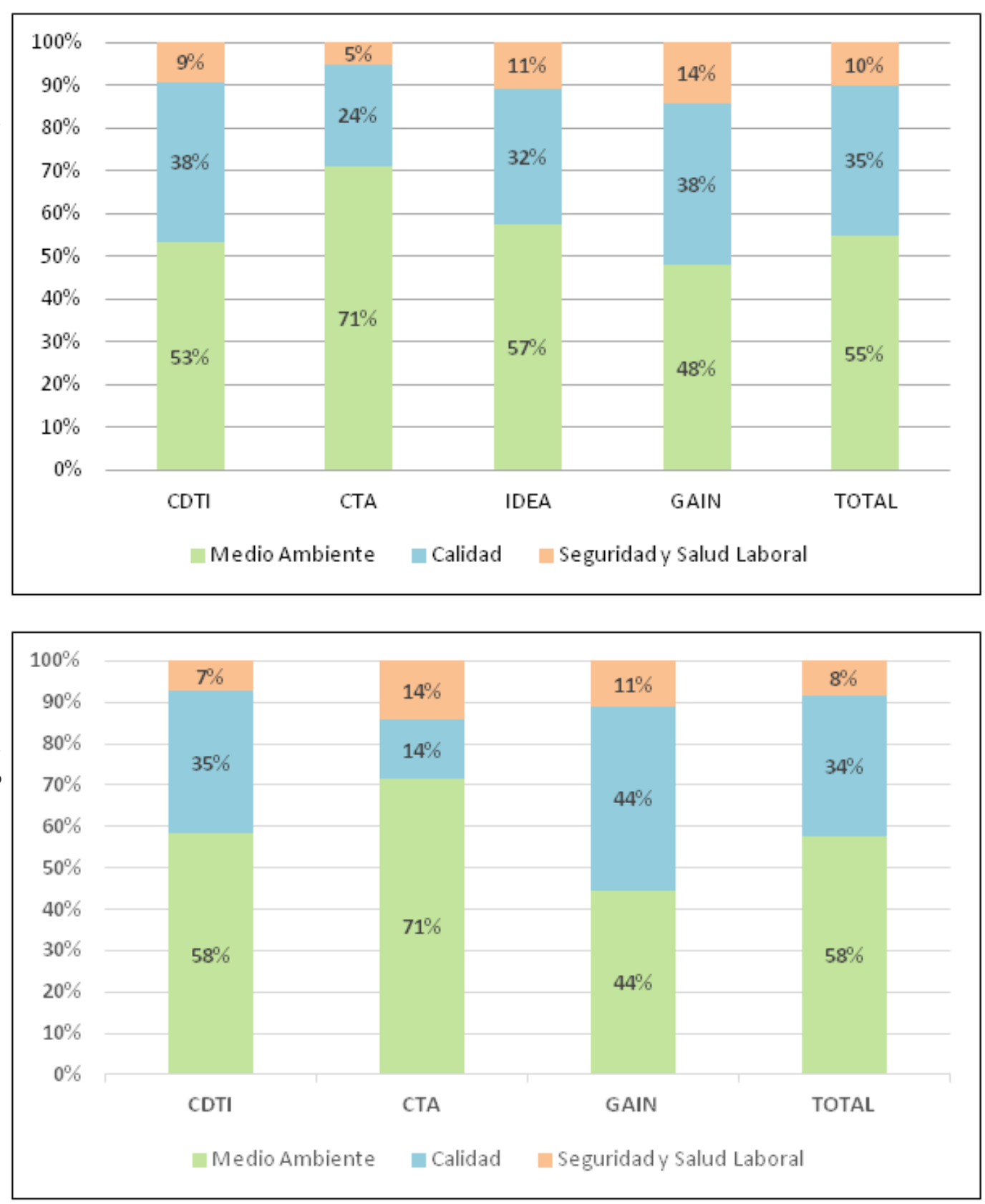

Como puede observarse en las figuras 2 y 3, tanto en cooperación como de forma individual, el porcentaje mayor de proyectos de $\mathrm{I}+\mathrm{D}+\mathrm{i}$ desarrollados se enmarca en el área de Medio Ambiente, seguido de los proyectos focalizados a la Calidad, clasificándose en último caso los proyectos centrados en la Seguridad y Salud Laboral. En el caso de la figura 3 no aparece la Agencia IDEA, ya que no financió ningún proyecto en cooperación.

\subsection{Resultados del estudio de casos}

Iniciamos este apartado describiendo las 3 empresas, para finalmente analizar los resultados comparados de las 3 empresas.

\section{Empresa 1: sector Energético}

Se trata de una empresa española con presencia internacional, con más de 70 años de trayectoria profesional, que ha crecido de forma progresiva hasta instaurar la innovación como una base importante de su actividad. Así, ha introducido una componente tecnológica que le aporta un valor añadido y le permite ser flexible ante los continuos cambios del mercado.

La empresa desarrolla, entre otras, varias líneas de actividad y negocio enfocadas a la creación de nuevas tecnologías con el objetivo principal de contribuir a un desarrollo sostenible. Su actividad está estructurada en las siguientes líneas de negocio: Solar, Bioenergía, Servicios Medioambientales, Tecnologías y Servicios de Información, Ingeniería y Construcción Industrial. 
La financiación de proyectos de $\mathrm{I}+\mathrm{D}+\mathrm{i}$, según las fuentes consultadas, se obtiene mediante dos mecanismos diferenciados y complementarios. Por un lado, financiación propia procedente de la compañía, y por otro mediante los programas de ayudas y subvenciones públicas existentes tanto en España como de origen internacional, principalmente en la Unión Europea y EEUU.

Los principales programas de ayuda a la innovación y las principales financiaciones públicas que han incentivado los proyectos de $\mathrm{I}+\mathrm{D}+\mathrm{i}$ de la empresa son el Programa CENIT y otros programas del CDTI, programas Marco de la EU y Horizonte 2020, así como la financiación del Departamento de Energía de EE.UU.

Tanto las líneas de investigación como los resultados obtenidos en los proyectos de $\mathrm{I}+\mathrm{D}+\mathrm{i}$ son aspectos protegidos y sólo difunden parte de la información según el interés y estrategia global de la empresa.

En 2010 se constituyó una empresa filial para fomentar y desarrollar proyectos de $\mathrm{I}+\mathrm{D}+\mathrm{i}$, con interés principal en los ámbitos de bioenergía, solar y Tecnologías de la Información y Comunicación (TIC). Su misión es recoger toda la actividad en $\mathrm{I}+\mathrm{D}+\mathrm{i}$, con carácter altamente innovador y actuar como vivero de ideas para la empresa matriz, generando nuevas opciones de futuro.

La mayor parte de la inversión en $\mathrm{I}+\mathrm{D}+\mathrm{i}$ se destina a la investigación aplicada y al desarrollo de innovación tecnológica, todo ello orientado a la obtención de los objetivos estratégicos de sostenibilidad y nuevos productos.

La $\mathrm{I}+\mathrm{D}+\mathrm{i}$ ha tenido en esta empresa un objetivo claro para el incremento de competitividad en las actividades de la empresa, contando con un Centro Tecnológico propio y colaboración continuada con Grupos de Investigación de Universidades. Cuentan con menos proyectos en Seguridad y Salud Laboral porque no se encuentra entre sus actividades principales. La empresa no difunde todas sus actividades de $\mathrm{I}+\mathrm{D}+\mathrm{i}$.

\section{Empresa 2: Sector de Obra civil}

Se trata de una empresa española con presencia internacional, que desarrolla actividades de edificación y obra civil, y presenta varias líneas de actividad y negocio, siendo la investigación e innovación un factor estratégico incorporado a su cultura empresarial como valor para garantizar la supervivencia de la organización y seguir construyendo una compañía de futuro. Desarrolla actividades en todas las áreas de construcción especialmente ferrocarril, alta velocidad, autovías y carreteras, puertos, aeropuertos, hidráulicas y medioambientales, tanto a nivel nacional como internacional.

Dispone de sistema de gestión integrado en los ámbitos de Calidad, Medio Ambiente y Seguridad y Salud Laboral, así como de I+D+i. El Sistema de Gestión de I+D+i se desarrolla conforme a la Norma UNE 166002.

La financiación para el desarrollo de los proyectos de $\mathrm{I}+\mathrm{D}+\mathrm{i}$, se ha obtenido mediante dos fuentes diferenciadas y complementarias. Por un lado, financiación propia de la compañía, y por otro, mediante los programas de ayudas y subvenciones públicas existentes en España, a través de las entidades públicas (CDTI y Agencia IDEA) y privada (CTA).

Tanto las líneas de investigación como los resultados obtenidos en los proyectos de $\mathrm{I}+\mathrm{D}+\mathrm{i}$ son aspectos protegidos y sólo difunden parte de la información según el interés y estrategia global de la empresa, fundamentalmente como herramienta de marketing e imagen social.

La empresa encara la I+D+i como un instrumento para el incremento de su competitividad y por diversificación de sus líneas de negocio. Cuenta con un departamento específico de I+D+i, y colabora de forma continuada con Centros de Investigación y Grupos de Investigación de Universidades. La empresa desarrolla menos proyectos en Seguridad y Salud Laboral que del resto de actividades y lo hace normalmente sólo por necesidades específicas.

\section{Empresa 3: Tecnologías de la Información y la Comu- nicación}

Se trata de una empresa española cuyo ámbito principal de actuación es la creación y aplicación empresarial de nuevas tecnologías. Desde sus inicios la creación de tecnología ha sido una de sus máximas en su estrategia empresarial. La tecnología que desarrolla puede enmarcarse en los siguientes campos: transporte y tráfico, energía, industria y consumo, administraciones públicas, sanidad, finanzas, seguros, seguridad y defensa, y telecomunicaciones. La empresa tiene fuerte presencia internacional, no sólo en Europa, sino también en Latinoamérica.

Una de las premisas que marcan su estrategia en el mercado es la competitividad y el valor añadido que introduce la tecnología que desarrolla. Su estrategia de innovación se basa en un modelo abierto que aprovecha tanto el talento interno (empleados) como el talento externo accesible a través de las relaciones con clientes exigentes, con socios y aliados líderes en sus respectivos nichos de actividad, con proveedores, con universidades e instituciones del conocimiento y con la sociedad en general.

Los proyectos se acogen a diferentes programas de financiación convocados por CDTI. A nivel internacional es la Comisión Europea la que financia los proyectos, concretamente a través del Programa FP7 y Horizonte 2020. Tanto las líneas de investigación como los resultados obtenidos en los proyectos de $\mathrm{I}+\mathrm{D}+\mathrm{i}$ son aspectos protegidos y sólo difunden parte de la información según el interés y estrategia global de la empresa. 
La I+D+i se utiliza como instrumento para el incremento de la competitividad y para adecuarse a las necesidades del cliente. La empresa está estructurada como Grupo de empresas. Cada empresa del Grupo desarrolla sus propias actividades de I+D+i. El Grupo cuenta con una Fundación específica para dar servicio de actividades de $\mathrm{I}+\mathrm{D}+\mathrm{i}$ y con la colaboración, también continuada, con Grupos de Investigación de Universidades. Igualmente desarrolla menos proyectos en Seguridad y Salud Laboral, y solo los desarrollan por requerimientos de demanda de mercado.

\subsection{Análisis del estudio de casos y de la infor- mación publicada de proyectos}

En los tres casos estudiados la organización para el desarrollo de actividades de $\mathrm{I}+\mathrm{D}+\mathrm{i}$ es diferente: Empresa filial del Grupo que centraliza todas las actividades de $\mathrm{I}+\mathrm{D}+\mathrm{i}$, Departamento específico de $\mathrm{I}+\mathrm{D}+\mathrm{i}$ y actividades de $\mathrm{I}+\mathrm{D}+\mathrm{i}$ desarrolladas de forma independiente por cada empresa del Grupo incluyendo una Fundación para proveer de servicios de $\mathrm{I}+\mathrm{D}+\mathrm{i}$. La estrategia de $\mathrm{I}+\mathrm{D}+\mathrm{i}$ también tiene aspectos diferenciadores en cada caso, desde la innovación en las actividades principales de la empresa, a la diversificación de líneas de negocio o la investigación para adecuarse a las necesidades del cliente.

No obstante, todas las empresas estudiadas desarrollan actividades de $\mathrm{I}+\mathrm{D}+\mathrm{i}$, tanto individuales como en cooperación, para incrementar su competitividad empresarial. En relación a los proyectos desarrollados en cooperación, éstos tienen un alcance mucho mayor que los que no lo son y principalmente son desarrollados para adecuarse a los programas de incentivo de los organismos públicos. Además, sirven para el desarrollo de nuevos contactos empresariales y el mantenimiento de la vigilancia tecnológica en el ámbito del sistema de gestión de I+D+i. Con carácter general, todas las empresas coinciden en señalar que los proyectos en colaboración permiten ampliar el alcance del proyecto y conseguir resultados más competitivos e innovadores. Desarrollar proyectos en consorcio, permite obtener mayor financiación pública, lo que se alinea con las aportaciones de Santamaría y Rialp (2007). A la vez, dichos proyectos presentan objetivos más ambiciosos frente a los que se realizan de forma individual (Santamaría y Rialp, 2007). Normalmente a la colaboración se le otorga un valor especial, pues favorece en general los ratios de éxito de los proyectos de I+D+i (Barajas y Huergo, 2006; Blindenbach-Driessen y Van Den Ende, 2006; Buesa et al., 2006; Hottenrott y Lopes-Bento, 2014; Kingsley et al., 1996; Santamaría y Rialp, 2007). De este modo, la cooperación se encara para incrementar la competitividad empresarial, lo que subraya lo obtenido por Bohnstedt et al. (2011), en cuanto a que los resultados de la inversión en proyectos de $\mathrm{I}+\mathrm{D}+\mathrm{i}$ elevan la productividad media de las empresas en el mercado. Estos proyectos también favorecen el desarrollo de la capacidad tecnológica para generar innovaciones (Belderbos et al., 2014). En cuanto al criterio de desarrollo de proyectos de $\mathrm{I}+\mathrm{D}+\mathrm{i}$ en cooperación, en cada caso depende de la estrategia de distribución del conocimiento y de las necesidades de incorporar conocimiento externo al desarrollo del proyecto.

Con respecto a los proyectos que desarrollan de forma individual, las empresas suelen realizarlos principalmente con el objetivo de proteger los resultados y líneas de investigación de la empresa. Incluso se realizan sin financiación externa para no publicitar o difundir estas actividades. Sin embargo, en algunos casos, la difusión de resultados es una herramienta de marketing y de acceso a nuevos mercados.

En todo caso, las empresas desarrollan las actividades de $\mathrm{I}+\mathrm{D}+\mathrm{i}$ con financiación propia conjuntamente con el incentivo de organismos de apoyo y fomento de la $\mathrm{I}+\mathrm{D}+\mathrm{i}$ públicos y privados. La financiación de las actividades de $\mathrm{I}+\mathrm{D}+\mathrm{i}$ es esencial para poder desarrollarlas, dado que se trata de proyectos con alto nivel de incertidumbre y riesgo de cara a obtener los resultados inicialmente previstos en el propio proyecto de I+D+i. En este sentido, las empresas consideran importante contar con incentivos suficientes en materia de Seguridad y Salud Laboral para poder desarrollar estas actividades.

Así, en todos los casos las empresas desarrollan proyectos de I+D+i en el ámbito de la Seguridad y Salud Laboral, tanto de forma individual como en cooperación. Quizás de forma significativa, todas las empresas disponen de sistema de gestión integrados de Calidad, Medio Ambiente y Seguridad y Salud Laboral, además de sistemas de gestión de I+D+i y de responsabilidad social corporativa. También en todos los casos, las empresas desarrollan menos proyectos de $\mathrm{I}+\mathrm{D}+\mathrm{i}$ en Seguridad y Salud Laboral que en materia de Calidad o de Medio Ambiente, ya sea por requerimientos de demanda de mercado, necesidades específicas, o directamente porque no están dentro de sus actividades principales. Destaca como en las tres empresas, en general, la Seguridad y Salud Laboral es una actividad secundaria frente a la Calidad o el Medio Ambiente, ya que en la mayoría de los casos no aparece esta temática en los proyectos o lo hace de forma colateral como requerimiento para otra actividad o temática principal del proyecto de $\mathrm{I}+\mathrm{D}+\mathrm{i}$. Al analizar la tendencia en el desarrollo de proyectos, de forma general se encontró que ésta era creciente. No obstante, en el caso particular de los proyectos de Seguridad y Salud Laboral, éstos se desarrollan a un ritmo más lento que los de Calidad o Medio Ambiente.

En relación a los aspectos clave para la investigación en Seguridad y Salud Laboral, la formación se considera crítica en este campo, tanto para los propios trabajadores y operarios como para los investigadores en éste ámbito. Sin una buena formación no se puede determinar el nivel de conocimiento en la materia y por tanto, tampoco se podrían generar ideas para el desarrollo de estos proyectos de I+D+i. Consecuentemente, al analizar el atractivo de unas temáticas frente a otras se encontró que la concienciación social en materia de Seguridad y Salud Laboral la consideran como un mínimo 
ya consolidado. Por ello, a pesar de que se considera inadmisible cualquier riesgo no controlado para los trabajadores, se percibe mayor recorrido en el caso del Medio Ambiente o la Calidad en cuanto a estos aspectos de concienciación y desarrollo de $\mathrm{I}+\mathrm{D}+\mathrm{i}$.

Este resultado se alinea con los resultados obtenidos al analizar los porcentajes de proyectos de $\mathrm{I}+\mathrm{D}+\mathrm{i}$ realizados en Medio Ambiente, Calidad y Seguridad y Salud Laboral por las tres empresas, los cuales están recogidos en las figuras 4 y 5 . Así, se encontró que los proyectos desarrollados en materia de Medio Ambiente y Calidad presentan una proporción muy superior a los de Seguridad y Salud. Además, destaca como los porcentajes de proyectos de Seguridad y Salud Laboral agregados para el caso de las tres empresas, son muy similares a los porcentajes obtenidos del estudio de la información publicada sobre la tipología de proyectos (véase figura 2 y 3 ). Concretamente, en el caso de proyectos de Seguridad y Salud Laboral desarrollados en cooperación, los tres casos agregados se sitúan ligeramente por debajo de la media general (véase figura 3 ) y en el caso de los desarrollados individualmente, están ligeramente por encima (véase figura 2). Por otro lado, resulta significativo el caso de la Empresa 1, ya que los proyectos de I+D+i en Seguridad y Salud Laboral son muy escasos en relación al total de proyectos desarrollados, tanto en cooperación como individualmente. Además, el porcentaje de proyectos de Medio Ambiente, tanto en cooperación como individuales, están muy por encima del porcentaje de proyectos desarrollados por las otras dos empresas y por la media general obtenida en el apartado anterior (véanse figuras 2 y 3 ). Este resultado puede venir motivado por el hecho de que la mayoría de las líneas de negocio de la Empresa 1 están directamente vinculadas al medio ambiente y las energías renovables.
Figura 4 Distribución acumulada de proyectos de $\mathrm{I}+\mathrm{D}+\mathrm{i}$ individuales de Seguridad y Salud Laboral, Calidad y Medio Ambiente obtenida del Estudio de Casos, durante el período 2009 a 2014 (agregado para los 3 casos).
Figura 5 Distribución acumulada de proyectos de $\mathrm{I}+\mathrm{D}+\mathrm{i}$ en cooperación de Seguridad y Salud Laboral, Calidad y Medio Ambiente obtenida del Estudio de Casos, durante el período 2009 a 2014 (agregado para los 3 casos).
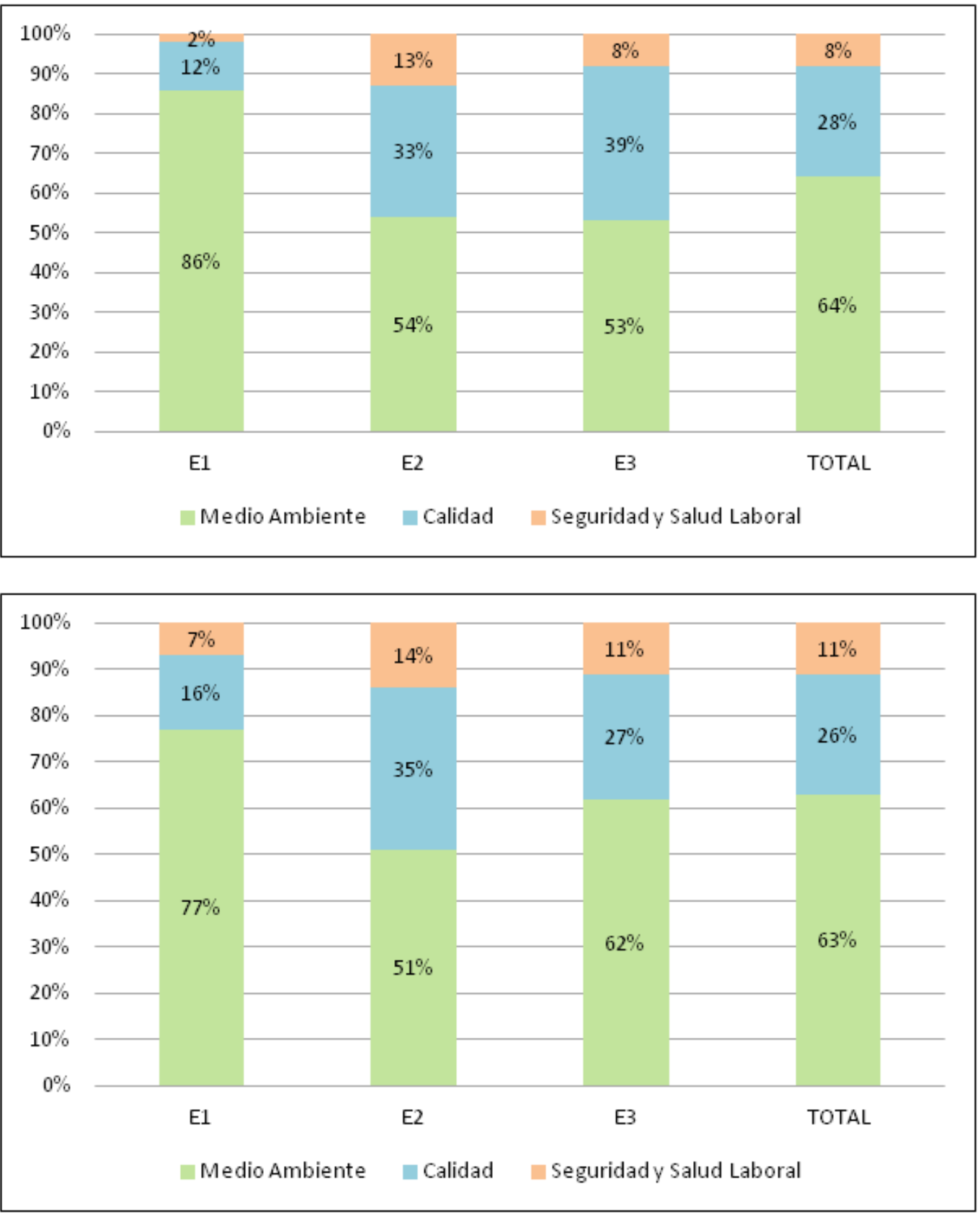


\section{Conclusiones}

En el periodo analizado, es decir, de 2009-2014, el CDTI era el organismo público con mayor número de proyectos gestionados, dado que era el principal organismo público de financiación de la $\mathrm{I}+\mathrm{D}+\mathrm{i}$ en España. Actualmente, comparte protagonismo con la Agencia Estatal de Investigación. Los organismos regionales, ya sean públicos o privados, en general, incentivaron proyectos de menor alcance económico que CDTI, ya que aunque gestionaron menos fondos el número de proyectos que incentivaron fue mayor proporcionalmente que en CDTI.

Además, en el análisis realizado se encontró que en el ámbito de la Seguridad y Salud Laboral el número de proyectos que se desarrollaron es menor que en las áreas de Calidad y Medio Ambiente. Al mismo tiempo, los proyectos de Seguridad y Salud Laboral eran dotados con una menor financiación respecto a los demás proyectos de las áreas mencionadas. Esto se traduce en una insuficiencia del fomento y apoyo a la $\mathrm{I}+\mathrm{D}+\mathrm{i}$ en Seguridad y Salud Laboral, lo que pone de relieve la necesidad promover nuevos programas de financiación, donde la Seguridad y Salud Laboral se constituya como una de las líneas prioritarias dada su transversalidad.

Como aspecto a mejorar hay que destacar que desde el diseño de las políticas públicas es necesario fomentar el incremento del número de proyectos de $\mathrm{I}+\mathrm{D}+\mathrm{i}$ desarrollados en cooperación en relación a los desarrollados individualmente. Sobre todo si se considera que la literatura pone de relieve que desarrollar proyectos en consorcio facilita oportunidades de obtener mayor financiación pública, y propician la definición de objetivos de I+D+i más ambiciosos, que los proyectos individuales. Sin embargo, en general, se incentivaron muchos más proyectos individuales que en cooperación. Particularmente, en el caso específico de los proyectos de I + D+i en Seguridad y Salud Laboral se observa una menor realización de proyectos en cooperación entre empresas que en el campo de Medio Ambiente o Calidad.

En relación a las limitaciones del estudio, por un lado esta investigación se ha centrado en los proyectos de Seguridad y Salud Laboral en comparativa con los ámbitos afines, de medio ambiente y la calidad. Sería de interés, por tanto, realizar estudios que abarcasen un número mayor de sectores/áreas donde se desarrolla I+D+i, para poder alcanzar una perspectiva más amplia. Sería también importante en este sentido disponer de repositorios públicos que facilitasen el acceso a esta información que permitiese su análisis. Por supuesto el estudio de casos presenta sus ventajas y sus inconvenientes. Entre estos últimos tenemos las dificultades de generalización, por lo que un estudio de inferencia estadística sería también deseable.

\section{Agradecimientos}

Los autores quieren agradecer a los responsables de las empresas que han participado su acceso y colaboración.

\section{Referencias bibliográficas}

Arroyabe, M. F., Arranz, N. \& de Arroyabe, J. C. F. (2015). R\&D partnerships: An exploratory approach to the role of structural variables in joint project performance. Technological Forecasting and Social Change, 90, 623634. https://doi.org/10.1016/j.techfore.2014.01.007

Barajas, A. \& Huergo, E. (2006). La cooperación tecnológica internacional en el ámbito de la empresa: una aproximación desde la literatura. Documentos de trabajo, CDTI, Dpto. de Estudios.

Belderbos, R., Cassiman, B., Faems, D., Leten, B. \& Van Looy, B. (2014). Co-ownership of intellectual property: Exploring the value-appropriation and value-creation implications of co-patenting with different partners. Research policy, 43(5), pp. 841-852. https://doi. org/10.1016/j.respol.2013.08.013

Blindenbach-Driessen, F. \& Van Den Ende, J. (2006). Innovation in project-based firms: The context dependency of success factors. Research policy, 35(4), pp. 545-561. https://doi.org/10.1016/j.respol.2006.02.005

Bohnstedt, A., Schwarz, C. \& Suedekum, J. (2011). Globalization and strategic research investments. Discussion paper series. Forschungsinstitut zur Zukunft der Arbeit. http://hdl.handle.net/10419/51676

Buesa, M., Heijs, J., Martínez, M. \& Baumert, T. (2006). Regional systems of innovation and the knowledge production function: the Spanish case. Technovation, 26 (4), pp. 463-472. https://doi.org/10.1016/j.technovation.2004.11.007

Chaminade, C., Ortín, P.L.S. \& Escobar, C.G. (1999). En busca de una teoría sobre la medición y gestión de los intangibles en la empresa: una aproximación metodológica. Ekonomiaz: Revista vasca de economía, (45), pp. 188-213.

Chetty, S. (1996). The case study method for research in small-and medium-sized firms. International small business journal, 15(1), 73-85. 
Crow, M.M. (1988). Technology and knowledge transfer in energy R\&D laboratories: An analysis of effectiveness. Evaluation and Program Planning. 11(1), pp. 85-95. https://doi.org/10.1016/0149-7189(88)90037-7

Evangelista, R. \& Sirilli, G. (1998). Innovation in the Service Sector Results from the Italian Statistical Survey. Technological Forecasting and Social Change. 58(3), pp. 251269. https://doi.org/10.1016/S0040-1625(98)00025-0

Freeman, C. \& Soete, L. (2009). Developing science, technology and innovation indicators: What we can learn from the past. Research policy, 38(4), pp. 583-589. https://doi.org/10.1016/j.respol.2009.01.018

García, M. \& Suárez, M. (2013). El método Delphi para la consulta a expertos en la investigación científica. Revista Cubana de Salud Pública. 39(2), pp. 253267. http://scielo.sld.cu/scielo.php?script=sci arttext\&pid=S0864-34662013000200007

Greenhalgh, C. \& Rogers, M. (2010). Innovation, intellectual property, and economic growth. Princeton University Press.

Hammadou, H., Paty, S. \& Savona, M. (2014). Strategic interactions in public R\&D across European countries: A spatial econometric analysis. Research policy, 43(7), pp. 1217-1226. https://doi.org/10.1016/j.respol.2014.01.011

Hottenrott, H. \& Lopes-Bento, C., (2014). (International) R\&D collaboration and SMEs: The effectiveness of targeted public R\&D support schemes. Research policy, 43(6), pp. 1055-1066. https://doi.org/10.1016/j.respol.2014.01.004

Kingsley, G., Bozeman, B. \& Coker, K., (1996). Technology transfer and absorption: an 'R \& D value-mapping' approach to evaluation. Research policy, 25(6), pp. 967995. https://doi.org/10.1016/0048-7333(96)00890-6

Lhuillery, S. \& Pfister, E. (2009). R\&D cooperation and failures in innovation projects: Empirical evidence from French CIS data. Research policy, 38(1), pp. 45-57. https://doi.org/10.1016/j.respol.2008.09.002

Myro, R. \& Lopez, M. E. Á. (Eds.). (2016). Una nueva política industrial para España. Consejo Económico y Social de España.

OIT (2017). Introduction to Occupational Health and Safety. International Labour Organization. Available at: http:// www.itcilo.org/en/the-centre/programmes/workers-ac$\underline{\text { tivities }}$
Patton, M.Q. (2002). Qualitative research and evaluation methods. Sage publications, 3rd edition. California.

Pereiras, M.S. \& Huergo, E. (2006). La financiación de actividades de $\mathrm{I}+\mathrm{D}+\mathrm{i}$ : una revisión de la evidencia sobre el impacto de las ayudas públicas. CDTI. https://www.cdti.es/recursos/publicaciones/archivos/7396_211121112006133850.pdf

Quintana, C. \& Benavides, C. A. (2010). Relación tecnológica en los acuerdos de cooperación empresarial y generación de innovaciones. Cuadernos de Economía y Dirección de la Empresa. 13(45), pp. 43-67. https://doi. org/10.1016/S1138-5758(10)70023-2

Rial-González, E., Copsey, S., Paoli, P. \& Schneider, E. (2005). Priorities for Occupational Safety and Health Research in the EU-25. European Agency for Safety and Health at Work, Strassen, Luxembourg. https:// osha.europa.eu/en/tools-and-publications/publications/ reports/6805648

Santamaría, L., Barge-Gil, A. \& Modrego, A. (2010). Public selection and financing of $R \& D$ cooperative projects: Credit versus subsidy funding. Research policy, 39(4), pp. 549-563. https://doi.org/10.1016/j. respol.2010.01.011

Santamaría, L. \& Rialp, J. (2007). La elección del socio en las cooperaciones tecnológicas: Un análisis empírico. Cuadernos de Economía y Dirección de la Empresa. 10(31), pp. 67-95. DOI: 10.1016/S1138-5758(07)70083-X

Schlegelmilch, B.B. \& Chini, T. (2003). Knowledge transfer between marketing functions in multinational companies: a conceptual model. International Business Review. 12(2), pp. 215-232. https://doi.org/10.1016/ S0969-5931(02)00097-5

Seale, C. \& Silverman, D. (1997): "Ensuring rigour in qualitative research". European Journal of Public health. Vol. 7, 379-384.

Tether, B.S. (2002). Who co-operates for innovation, and why: An empirical analysis. Research policy, 31(6), pp. 947-967. https://doi.org/10.1016/S00487333(01)00172-X

Vives, X. (2008). Innovation and competitive pressure. Journal of Industrial Economics 56(3), pp. 419-469.

Yin, R. (2009). Case Study Research: Design and Methods, SAGE Publications. California, USA. 